\title{
Correction to: Top-down Modulation of Outcome Processing in Primary Sensory Cortex for Flexible Behavior
}

\author{
Qi Cheng ${ }^{1,2} \cdot$ Chengyu T. Li ${ }^{1,2}$
}

Published online: 9 May 2021

(C) Center for Excellence in Brain Science and Intelligence Technology, Chinese Academy of Sciences 2021

\section{Correction to: Neurosci. Bull.} https://doi.org/10.1007/s12264-021-00656-1

The original version of this article unfortunately contained some mistakes.
The authors found that in this article, the Fig. 1 was wrong, and the corrected Fig. 1 was showed as following.

The original article can be found online at https:// doi.org/10.1007/s12264-021-00656-1.

Qi Cheng

qcheng@ion.ac.cn

$\triangle$ Chengyu T. Li

tonylicy@ion.ac.cn

1 Institute of Neuroscience, State Key Laboratory of Neuroscience, Chinese Academy of Sciences, CAS Center for Excellence in Brain Science and Intelligence Technology, Shanghai Center for Brain Science and Brain-Inspired Technology, Shanghai 200031, China

2 School of Future Technology, University of the Chinese Academy of Sciences, Beijing 100049, China 


\section{Rule switch}

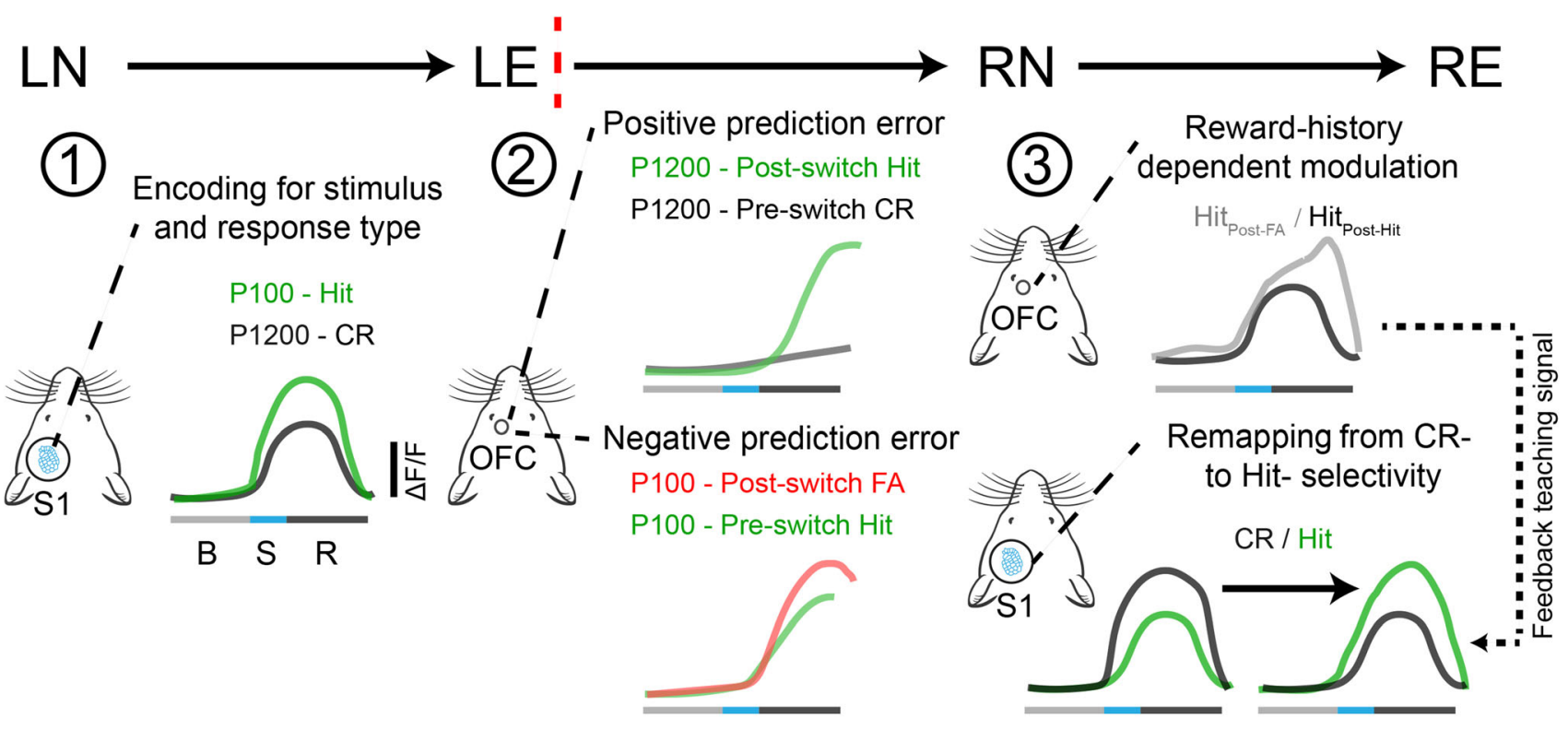

Fig. 1 Schematic showing the dynamic recruitment of S1 and 1OFC neurons and their interactions supporting the adaptive decisions during different learning phases of a reversal learning task, based on the findings of Banerjee et al. [6]. During initial learning (phase 1, from $\mathrm{LN}$ to $\mathrm{LE}$ ) of a texture-discrimination 'go/no-go task', S1 encodes both the sensory stimulus and the behavioral outcome, which is critical for mice to learn the S-O contingency. After the S-O contingency is switched, the 1OFC saliently and transiently encodes both positive and negative prediction errors after immediate reversal (phase 2, from LE to $\mathrm{RN}$ ), which contributes to the flexible remapping of S-O contingency. When mice re-learn the switched S-O contingency (phase 3, from RN to RE), reward-history-dependent signals in the IOFC facilitate the re-mapping from Correct-rejectionselective to Hit-selective activity in S1. B, baseline; S, stimuluspresentation window; $\mathrm{R}$, reward-outcome window. 